\title{
Nonlaxative PET/CT Colonography: Feasibility, Acceptability, and Pilot Performance in Patients at Higher Risk of Colonic Neoplasia
}

Stuart A. Taylor ${ }^{1,2}$, Jamshed B. Bomanji ${ }^{3}$, Levi Manpanzure ${ }^{1}$, Charlotte Robinson ${ }^{1}$, Ashley M. Groves ${ }^{3}$, John Dickson ${ }^{3}$, Nickolaos D. Papathanasiou ${ }^{3}$, Rebecca Greenhalgh ${ }^{1}$, Peter J. Ell ${ }^{3}$, and Steve Halligan ${ }^{1,2}$

${ }^{I}$ Department of Specialist Radiology, University College London Hospital NHS Trust, London, United Kingdom; ${ }^{2}$ Department of Medicine, University College London, London, United Kingdom; and ${ }^{3}$ Institute of Nuclear Medicine, University College London, London, United Kingdom

CT colonography without bowel preparation is a safer and bettertolerated alternative to full laxation protocols, but comparative sensitivity and specificity are potentially reduced. Uptake of ${ }^{18} \mathrm{~F}-\mathrm{FDG}$ by colonic neoplasia is well described, and combining PET with nonlaxative CT colonography could improve accuracy. The purpose was to prospectively test the technical feasibility and acceptability of combined nonlaxative PET/CT colonography in patients at higher risk of colorectal neoplasia and to provide pilot data on diagnostic performance. Methods: Fifty-six patients (median age, $64 \mathrm{y}$; 30 women) at high risk of colonic neoplasia underwent nonlaxative PET/CT colonography with barium fecal tagging within 2 wk of scheduled colonoscopy. Colonic segmental distension was graded 1 (poor) to 3 (good). A radiologist, experienced in CT colonography, and nuclear medicine physician in consensus analyzed the datasets. The diagnostic performance for standalone CT colonography and combined $\mathrm{PET} / \mathrm{CT}$ colonography was compared with the reference colonoscopy. Patient experience for 25 items (each scored from 1 to 7 ) pertaining to satisfaction, worry, and physical discomfort was canvassed after both PET/CT colonography and colonoscopy. Results: Distension was good in 298 of 334 segments (89\%; 95\% confidence interval [Cl], 85\%-92\%). Patients experienced more physical discomfort during colonoscopy (median, 4; interquartile range [IQR], 2-7) than during PET/CT colonography (median, 5; IQR, 3-7; $P=0.03$ ) and were more willing to undergo PET/CT colonography again (36/43 [84\%; 95\% Cl, 73\%-95\%] vs. $31 / 43$ [72\%; 95\% Cl, 59\%-86\%]; $P=0.001)$. Twenty-one patients had 54 polyps according to colonoscopy (10 with at least 1 polyp $\geq 6 \mathrm{~mm}$ and 8 with at least 1 polyp $\geq 10 \mathrm{~mm}$ ). Of 14 polyps $6 \mathrm{~mm}$ or greater, 12 (86\%; 95\% Cl, 67\%-100\%) were ${ }^{18} \mathrm{~F}-\mathrm{FDG}$-avid, including all those $10 \mathrm{~mm}$ or greater (mean standardized uptake value, 10.1). CT colonography sensitivity for polyps $6 \mathrm{~mm}$ or larger was $92.9 \%(95 \% \mathrm{Cl}, 79.4 \%-100 \%)$ and was not improved by the addition of PET. However, combined PET/CT colonography review improved per-patient positive predictive value for a polyp $10 \mathrm{~mm}$ or greater from $73 \%$

Received Nov. 11, 2009; revision accepted Mar. 1, 2010.

For correspondence or reprints contact: Stuart Taylor, Department of Specialist Imaging, University College Hospital, 2F Podium, 235 Euston

Rd., London, U.K., NW1 2BU.

E-mail: csytaylor@yahoo.co.uk

COPYRIGHT ( 2010 by the Society of Nuclear Medicine, Inc.
(95\% Cl, 39-92) to $100 \%(95 \% \mathrm{Cl}, 60-100)$. Conclusion: In this feasibility study, simultaneous PET acquisition during nonlaxative CT colonography is technically feasible, is well tolerated, and potentially improves specificity.

Key Words: colonic polyps/diagnosis; colonography; computed tomographic/methods; feasibility studies; fluorodeoxyglucose ${ }^{18} \mathrm{~F} /$ diagnostic use

J Nucl Med 2010; 51:854-861

DOI: 10.2967/jnumed.109.072728

\section{$\mathrm{C}$}

$\mathrm{T}$ colonography has proven reliable for the diagnosis of colorectal neoplasia (1). Implementation is particularly attractive in symptomatic older patients who are at increased risk of complications from colonoscopy (2) but in whom-despite symptom triage-the incidence of colonic cancer is at the most $10 \%$ (3). There is evidence, however, that arduous bowel purgation not only is detrimental to compliance (4) but also may cause serious adverse effects including significant electrolyte imbalance (5). Considerable interest exists in reducing or even eliminating laxation from CT protocols by adding positive oral contrast agents to patients' diet to label or tag residual stool as higher attenuation to aid radiologist interpretation. Although data from such fecal-tagging protocols are promising, both sensitivity and specificity may be below that of conventional CT colonography, even for large colonic lesions $(6,7)$. Results using iodine-based tagging regimens rather than barium may be superior $(8,9)$ but also remain under investigation.

Uptake of ${ }^{18}$ F-FDG by colonic adenomas and cancer has been described during PET $(10,11)$. If colonic neoplasia in laxative-free CT colonography were reliably highlighted via ${ }^{18}$ F-FDG avidity, combined PET/CT colonography might have the potential to be a safe, well-tolerated, and accurate 
diagnostic test for clinically significant colorectal neoplasia in high-risk symptomatic patients. Although the technical feasibility of combined PET/CT colonography has been described previously (12-15), most studies have used small cohorts and patients have undergone full purgation.

The purpose of our study was to prospectively test the technical feasibility and patient acceptability of nonlaxative PET/CT colonography for the diagnosis of colorectal neoplasia in older patients at higher risk and to provide pilot data on diagnostic performance.

\section{MATERIALS AND METHODS}

Ethical permission was obtained from the ethics committee of University College Hospital London in 2007, and informed written consent was obtained from all patients.

Between April 2007 and April 2009, patients older than 45 y undergoing colonoscopy as part of routine clinical care were invited to undergo nonlaxative PET/CT colonography during the 2 wk before their colonoscopy. Review of the colonoscopy referral letter allowed us to recruit patients at high risk of colorectal neoplasia - notably those patients who had a significant change in bowel habit, who were undergoing polyp surveillance, who had a family history of colorectal cancer, or who had a positive fecal occult blood test. Patients who were pregnant or had a known diagnosis of inflammatory bowel disease (known to itself produce increased colonic ${ }^{18}$ F-FDG uptake) were excluded.

One hundred twenty-five patients were approached, and 58 agreed to participate. Two patients were excluded (1 technical failure and 1 patient withdrawal), leaving 56 patients (median age, 64 y; age range, 46-83 y; 30 women). Nineteen were undergoing colonoscopy because of fecal occult blood; 13 because of a change in bowel habit; 11 and 8 because of a family or personal history of colorectal cancer, respectively; and 5 because of rectal bleeding.

\section{Procedures}

PET/CT Colonography. The day before PET/CT colonography, patients were instructed to follow a low-fiber diet. In particular, whole-grain cereal and vegetables were excluded. With each of the 3 meals, patients also consumed $20 \mathrm{~mL}$ of $40 \% \mathrm{w} / \mathrm{v}$ barium sulphate (Tagitol V; a kind gift of Bracco Diagnostics, Ltd.) to tag residual stool. No laxatives were given. Patients were able to drink water on the morning of PET/CT colonography.

After confirmation of a blood glucose level less than $8 \mathrm{mmol} / \mathrm{L}$, patients were injected with $185 \mathrm{MBq}$ of ${ }^{18} \mathrm{~F}-\mathrm{FDG}$ (half of the normal clinical dose to reduce radiation exposure [effective dose, $4.75 \mathrm{mSv}]$ ) and $1 \mathrm{~h}$ later were transferred onto the PET/CT scanner table (Discovery DST16; GE Healthcare). Twenty milligrams of hyoscine butylbromide (Buscopan; Boehringer Ingelheim Ltd.) were administered intravenously, and colonic insufflation commenced using an automated $\mathrm{CO}_{2}$ insufflator (ProtocOL; Bracco Diagnostics Ltd.). Insufflation continued until the practitioner considered the colon was likely to be adequately distended-distension was based on reported patient feelings of bloating and confirmed with a CT scout scan (16) (mean insufflated volume, $3.1 \mathrm{~L}$; range, 2.2 to $4.1 \mathrm{~L}$ ). Supine CT data were then acquired (1.25-mm collimation; $140 \mathrm{kVp}$; and $80 \mathrm{~mA}$ for body mass index $[\mathrm{BMI}]<25,100 \mathrm{~mA}$ for BMI of 25-35, and $120 \mathrm{~mA}$ for $\mathrm{BMI}>$ 35 without dose modulation; $0.8 \mathrm{~s}$; and 1.75 pitch). Supine PET acquisition was subsequently performed using 3 bed positions to encompass the colon and an 8-min acquisition per position.
During PET acquisition, colonic distension was maintained by low-pressure automated insufflation, with the pressure dial set at $15 \mathrm{~mm} \mathrm{Hg}$. CT colonography and PET data were then acquired with the patient prone, in the same way as for supine acquisition, after administration of a further $20 \mathrm{mg}$ of hyoscine butylbromide and additional gas insufflation (mean insufflated volume, $500 \mathrm{~mL}$ ) if the prone CT scout scan suggested colonic distension was suboptimal. The total examination time was $55 \mathrm{~min}$. All patients had a BMI less than 35 . The effective doses for the CT component of the examination were $3.8 \mathrm{mSv}(80 \mathrm{~mA})$ or $4.8 \mathrm{mSv}(100 \mathrm{~mA})$ for men or $4.8 \mathrm{mSv}(80 \mathrm{~mA})$ or $6.0 \mathrm{mSv}(100 \mathrm{~mA})$ for women (CT Expo, version 1.6(E), radiation dose calculation, Stamm and Nagel, Hannover/Buchholz, 2001-2007).

Imaging Processing. Combined PET/CT data were reconstructed using ordered-subset expectation maximization, with 2 iterations and 21 subsets and a $128 \times 128$ matrix using an axial field of view of $70 \mathrm{~cm}$ and a slice thickness of $3.27 \mathrm{~mm}$ (pixel size, $5.47 \mathrm{~mm}[700 / 128])$. The CT image was reconstructed with a slice thickness of $1.25 \mathrm{~mm}(50-\mathrm{cm}$ field of view, $512 \times 512$ matrix, and 0.98-mm pixel size).

CT Colonography Technical Quality. Two radiologists (with 3 and $4 \mathrm{y}$ of CT colonography experience and more than 400 examinations) reviewed each of the CT colonography datasets on a segmental basis and assigned a score for distension quality (1, poor; 2 , adequate; and 3 , good) and adequacy of fecal tagging (1, all residual stool untagged; $2,<25 \%$ of residual stool adequately tagged; $3, \geq 25 \%-50 \%$ of residual stool adequately tagged; and $4,>50 \%$ of residual stool adequately tagged).

Image Analysis. CT colonography datasets were interpreted by a radiologist with experience with more than 1,000 validated CT colonography datasets, unaware of the PET component and clinical history. A primary 2-dimensional reading paradigm on a PACS (Agfa-Gevaert Group), equipped with full multiplanar reformat ability, was used. A 3-dimensional problem-solving volume-rendered cube was available if required via dataset export to a CT colonography workstation (Vitrea 4.2; Vital Images). Using standard criteria (17), the radiologist searched for colonic neoplasia and noted the longest 2-dimensional measurement and colonic segmental location, assigning a score of 1 (least confident) to 4 (certain) indicating confidence that the abnormality was real. The radiologist was, however, told all reported lesions would be included in the comparison to colonoscopy. The radiologist also assigned a score indicating confidence that a lesion $10 \mathrm{~mm}$ or greater could be excluded from those colonic segments thought to be normal (score: 1, cannot exclude; 2 , difficult to exclude; 3 , probably exclude; and 4, definitely exclude).

\section{PET/CT Colonography}

The radiologist reading the CT colonography images then interpreted the fused PET/CT images in consensus with a nuclear medicine physician (7 y of experience with PET/CT reporting). Colonic pathology on PET was defined according to the criteria of Gollub et al. (12) as a focal area of increased ${ }^{18} \mathrm{~F}-\mathrm{FDG}$ uptake over and above that of background colonic activity. Diffuse curvilinear ${ }^{18}$ F-FDG uptake was assumed to represent normal bowel activity. No specific threshold standardized uptake value (SUV) was used for identifying a colonic abnormality.

The avidity or otherwise of all CT colonography-detected lesions was estimated, and a consensus decision made as to whether (based on all imaging findings) the lesion was real or a CT false-positive (e.g., untagged residual stool). The SUV of all 
lesions deemed to be real (if PET-avid) was recorded on the supine dataset, and any apparent misregistration between the CT abnormality and area of PET avidity in the fused dataset was measured using the most appropriate multiplanar reconstruction. Those segments originally assigned a confidence score of 2 or less for exclusion of a polyp $10 \mathrm{~mm}$ or smaller were reviewed, and a revised consensus confidence score based on the distribution of ${ }^{18} \mathrm{~F}-\mathrm{FDG}$ activity within the segment was assigned. Any extracolonic abnormalities were also noted using both the CT and PET image components.

\section{Colonoscopy}

Within 2 wk of PET/CT colonography, patients underwent colonoscopy undertaken by experienced endoscopists ( $\geq 5 \mathrm{y}$ ) after full bowel preparation (13 $\mathrm{g}$ of senna granules [Reckitt Benckiser Healthcare] and $35 \mathrm{~g}$ of magnesium citrate [Citramag; Sanochemia]). Sedation (median, $1.25 \mathrm{mg}$ of midazolam; Phoenix Pharma Ltd.) and analgesia (median, $25 \mathrm{mg}$ of pethidine [Pamergan P100]; Martindale Pharmaceuticals Ltd.) were administered as required. Endoscopists were aware of the consensus PET/CT report-so all reported imaging findings could be confirmed or refuted by the reference colonoscopy - and recorded the colonic segment of detected polyps, estimating diameter by direct comparison to adjacent open biopsy forceps. Histologic examination was performed by a group of local histopathologists using standard criteria (18). In particular, high-grade dysplasia was defined as complex glandular crowding and irregularity with cribiform appearance and back-to-back glands, prominent intraluminal papillary tufting, and marked glandular budding.

\section{Polyp Matching}

The study coordinator ( 3 y of experience with CT colonography and more than 200 examinations) reviewed the PET/CT colonography and colonoscopy reports and applied published matching criteria (lesions within 1 colonic segment and size within 50\%) (19) to determine the sensitivity and specificity of CT colonography alone and then of combined PET/CT colonography, compared with the reference colonoscopy. All polyps identified at colonoscopy but unreported by the study radiologist were evaluated in retrospect to see if they were visible.

Patient Experience. A questionnaire, previously validated for patients undergoing colonoscopy (20), was administered to patients after both the PET/CT colonography and the colonoscopy (Supplemental Appendix 1; supplemental materials are available online only at http://jnm.snmjournals.org). Patients completed the questionnaires at home on the day after each test.

A second questionnaire (Supplemental Appendix 2) investigating overall preference between the 2 tests was administered; patients completed this questionnaire at home $48 \mathrm{~h}$ after colonoscopy (in an attempt to reduce bias induced by the initial postcolonoscopy recovery). The questionnaire included a $10-\mathrm{cm}$ visual analog scale between "the bowel preparation for PET/CT was best" and "the bowel preparation for colonoscopy was best."

\section{Statistical Analysis}

The proportion of segments assigned a confidence score of 2 or less for exclusion of a lesion $10 \mathrm{~mm}$ or larger was compared between CT colonography alone and combined PET/CT using the Fisher exact test. SUV was compared between adenomas with and without high-grade dysplasia using an unpaired $t$ test. Per-patient sensitivity and specificity were compared between CT colonography alone and combined PET/CT using a paired exact test.

\section{Patient Questionnaires}

The Wilcoxon matched pairs test was used to compare responses after grouping the data into 3 principal components (physical discomfort, worry, and satisfaction; Supplemental Appendix 1). Patients tolerating the procedures well and the number willing to repeat the procedure were compared using a binomialbased exact test. A comparison of which procedure was preferred and most acceptable was made using a paired analysis of proportions.

\section{RESULTS}

No complications were reported after either PET/CT colonography or colonoscopy.

\section{CT Colonography Technical Quality}

Three hundred thirty-four colonic segments were assessed (1 patient had a right hemicolectomy). The mean segmental colonic distension score was 2.88 (SD, 0.36; median, 3; and interquartile range [IQR], 3-3). In total, 298 of 334 segments (89\%; 95\% confidence interval [CI], $85 \%-92 \%$ ) achieved a distension score of 3 .

Tagging quality was also high, with a mean tagging score of 3.69 (SD, 0.86; median, 4; and IQR, 4-4). In total, 289 of 334 segments (87\%; 95\% CI, 83\%-90\%) achieved a tagging quality score of 4 . Of those 39 segments scoring less than 4, $27(70 \%$; 95\% CI, 55\%-84\%) were in the rectum or sigmoid.

\section{Diagnostic Performance}

Of the 56 patients, 34 patients had normal results on colonoscopy and 1 patient had right-sided Crohn colitis discernable as colonic wall thickening and diffuse abnormal ${ }^{18}$ F-FDG uptake. The remaining 21 patients harbored a total of 54 polyps (40 polyps, $1-5 \mathrm{~mm}$; 4 polyps, $6-9 \mathrm{~mm}$; and 10 polyps, $\geq 10 \mathrm{~mm}$ ). Of the 1 - to 5 -mm polyps, 16 were not retrieved for histology, 9 were hyperplasic, and 15 were adenomas (12 tubular and 3 tubulovillous). Of the 6 mm or larger polyps, 12 were adenomatous (2 tubular and 10 tubulovillous), of which 5 showed high-grade dysplasia, 1 was a hemangioma, and 1 was hyperplastic. Ten patients harbored at least 1 polyp $6 \mathrm{~mm}$ or larger, and 8 at least 1 polyp $10 \mathrm{~mm}$ or larger. No patient had cancer.

The diagnostic performance of CT colonography and combined PET/CT colonography is shown in Tables 1 and 2. A nonreported 6-mm polyp was not visible, even in retrospect, because of poor segmental stool tagging, although an additional 7 polyps $(1-5 \mathrm{~mm})$ not reported by the study radiologists were visible on retrospective unmasked review.

Combined PET/CT colonography did not improve the sensitivity of standalone CT colonography. Of 14 polyps $6 \mathrm{~mm}$ or larger, $12\left(86 \%\right.$; 95\% CI, 67\%-100\%) were ${ }^{18} \mathrm{~F}-$ FDG-avid (including all those $\geq 10 \mathrm{~mm}$ ), with a mean SUV of 10.1 (range, 3.4-22) (Figs. 1 and 2). There was no significant difference in mean SUV for adenomas with (mean, 12.7) and without (mean, 7.4) high-grade dysplasia $(P=$ 0.10). The single 6-mm polyp missed on CT colonography 
TABLE 1. Sensitivity of Nonlaxative CT Colonography and Combined PET/CT Colonography for Diagnosis of Colonic Neoplasia (per Polyp)

\begin{tabular}{|c|c|c|c|c|c|c|c|c|c|c|}
\hline \multirow[b]{2}{*}{ Polyp size (mm) } & \multirow[b]{2}{*}{ Total no. } & \multicolumn{3}{|c|}{ CT colonography detection } & \multicolumn{3}{|c|}{ PET/CT detection } & \multicolumn{3}{|c|}{${ }^{18} \mathrm{~F}-\mathrm{FDG}$ avidity } \\
\hline & & $n$ & $\%$ & $95 \% \mathrm{Cl}$ & $n$ & $\%$ & $95 \% \mathrm{Cl}$ & $n$ & $\%$ & $95 \% \mathrm{Cl}$ \\
\hline $1-5$ & 40 & 9 & 22.5 & $9.6-35$ & 9 & 22.5 & $9.6-35$ & 0 & 0 & \\
\hline $6-9$ & 4 & 3 & 75 & $33-100$ & 3 & 75 & $33-100$ & 2 & 50 & 10-99 \\
\hline$\geq 10$ & 10 & 10 & 100 & $100-100$ & 10 & 100 & $100-100$ & 10 & 100 & $100-100$ \\
\hline
\end{tabular}

was not ${ }^{18}$ F-FDG-avid (Fig. 3), nor was any polyp $5 \mathrm{~mm}$ or smaller.

There was perfect registration in 10 of the ${ }^{18} \mathrm{~F}-\mathrm{FDG}$-avid polyps. In one 20-mm sigmoid polyp, there was $11 \mathrm{~mm}$ of misregistration (Fig. 4) and in one 6-mm transverse polyp, $3 \mathrm{~mm}$ of misregistration. There were $9 \mathrm{CT}$ colonography false-positives $(7-12 \mathrm{~mm})$ in 6 patients, 5 of whom had no other true polyp $6 \mathrm{~mm}$ or larger. All were non- ${ }^{18} \mathrm{~F}-\mathrm{FDG}-$ avid and were correctly dismissed after combined PET/CT colonography review, improving per-patient specificity from $89 \%$ to $100 \%$ at the 6 -mm threshold $(P<0.01)$ and positive predictive value (PPV) from $73 \%$ to $100 \%$ at the 10-mm threshold (95\% CI 39\%-92\%) (Table 2; Fig. 5).

The mean confidence score was 3.37 (SD, 0.64; range, 2-4) for CT colonography true-positive detections and 2.13 (SD, 0.54; range, 1-3) for false-positive detections. In terms of diagnostic confidence, $7 \%$ (95\% CI, 4\%-9\%) of segments were assigned a score of 2 or less after CT colonography alone, improving to $1.2 \%$ (95\% CI, $0 \%-2.4 \%)$ with PET $(P<0.001)$.

Extracolonic Findings. Extracolonic findings were reported in 12 patients (excluding simple liver and renal cysts) and included large uterine fibroids (2 patients), gallstones ( 2 patients), gastric carcinoma, a presacral mass (little ${ }^{18} \mathrm{~F}$-FDG avidity and benign on work-up), renal obstruction ( 2 patients), an adrenal mass (non- ${ }^{18} \mathrm{~F}-\mathrm{FDG}-$ avid and benign on work-up), an enlarged prostate, and pancreatic atrophy (2 patients). All extracolonic findings, except the gastric carcinoma-which was detected via diffuse focal ${ }^{18} \mathrm{~F}$-FDG uptake on the combined PET/CT colonography dataset-were diagnosed on the $\mathrm{CT}$ component.

\section{Patient Experience}

Forty patients returned the questionnaire for both procedures (combined PET/CT colonography and colonoscopy). When items were grouped by principal component, there was no significant difference in overall patient worry (both procedures: median, 6; IQR, 4-7) or satisfaction (both procedures: median, 7; IQR, $6-7)$ for either procedure $(P=$ 0.55 and 0.77 , respectively). However, patients experienced significantly more physical discomfort during colonoscopy (median, 4; IQR, 2-7) than during PET/CT colonography (median, 5; IQR, 3-7) $(P=0.03)$.

Forty-three patients returned the final questionnaire pertaining to overall tolerance and preference. There was no significant difference in patient tolerance (Fig. 6; $P=$ 0.77). However, patients were more willing to undergo PET/CT colonography (36/43 [84\%]) again than colonoscopy $(31 / 43[72 \%])(P=0.001)$.

Patients preferred to undergo PET/CT colonography again rather than colonoscopy if they had a choice $(P=$ 0.002), and overall they found PET/CT colonography the more acceptable test $(P<0.001)$ (Fig. 7). Analysis of bowel preparation via the visual analog scale found that there was a strong preference for PET/CT colonography (mean place on 10-cm scale, $2.5 \mathrm{~cm}$; SD, $1.5 \mathrm{~cm}$; median, 2; and IQR, 2-3).

\section{DISCUSSION}

The main aim of our study was to test the technical feasibility and patient acceptance of nonlaxative PET/CT colonography. We confirmed that the procedure was technically feasible, with generally high-quality distension and

TABLE 2. Per-Patient Diagnostic Performance of Nonlaxative CT Colonography and Combined PET/CT Colonography for Lesions $\geq 6 \mathrm{~mm}$

\begin{tabular}{|c|c|c|c|c|c|c|c|c|c|}
\hline \multirow[b]{2}{*}{ Largest polyp (mm) } & \multirow[b]{2}{*}{ Total no. } & \multicolumn{2}{|c|}{ Sensitivity } & \multicolumn{2}{|c|}{ Specificity } & \multicolumn{2}{|c|}{$\begin{array}{c}\text { Negative } \\
\text { predictive value }\end{array}$} & \multicolumn{2}{|c|}{ PPV } \\
\hline & & $\%$ & $95 \% \mathrm{Cl}$ & $\%$ & $95 \% \mathrm{Cl}$ & $\%$ & $95 \% \mathrm{Cl}$ & $\%$ & $95 \% \mathrm{Cl}$ \\
\hline \multicolumn{10}{|l|}{ CT colonography alone } \\
\hline$\geq 6^{\star}$ & 10 & 100 & $100-100$ & 89.1 & $80-98$ & 100 & $89-100$ & 68 & $39-97$ \\
\hline$\geq 10^{+}$ & 8 & 100 & $100-100$ & 93.8 & $87-100$ & 100 & $90-100$ & 73 & 39-92 \\
\hline \multicolumn{10}{|l|}{ PET/CT colonography } \\
\hline$\geq 6$ & 10 & 100 & $100-100$ & 100 & $100-100$ & 100 & $90-100$ & 100 & $65-100$ \\
\hline$\geq 10$ & 8 & 100 & $100-100$ & 100 & $100-100$ & 100 & $90-100$ & 100 & $60-100$ \\
\hline $\begin{array}{l}{ }^{*} \text { Prevalence, } 18 \% . \\
{ }^{\dagger} \text { Prevalence, }-14 \% .\end{array}$ & & & & & & & & & \\
\hline
\end{tabular}



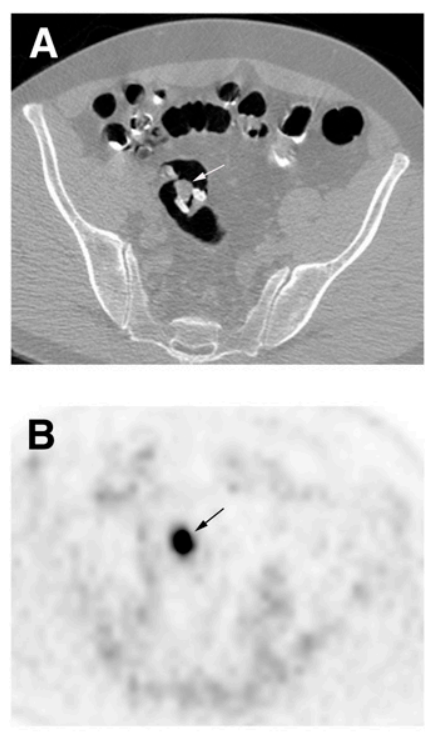

FIGURE 1. Axial CT (A), PET (B), and fused PET/CT colonography $(C)$ images demonstrating 20-mm sigmoid tubulovillous adenoma (arrow), which was ${ }^{18}$ F-FDG-avid (SUV, 22).

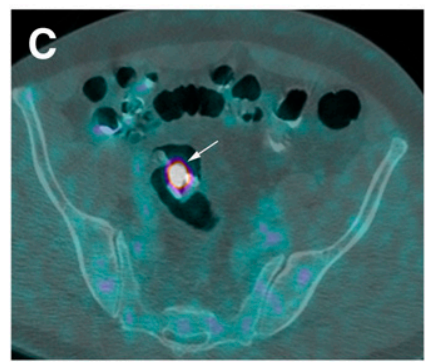

bowel preparation; this result was reassuring given the relatively prolonged examination time necessary for PET/ CT colonography in comparison to CT colonography alone. Our choice of carbon dioxide (which is absorbed by the colon) over air was governed by the superior patient tolerance of the former (21). The readers were confident that a lesion greater than $10 \mathrm{~cm}$ could be excluded in $98.2 \%$ of colonic segments using PET/CT colonography. Arguably, it would have been useful to also assess confidence in excluding a 6- to 9-mm polyp, but our focus was on those lesions that would be deemed clinically important in this older patient cohort.

We found that patients much preferred the minimal PET/ CT colonography bowel preparation and reported significantly more physical discomfort during colonoscopy, despite sedation and the long examination time of the PET/CT colonography itself $(55 \mathrm{~min})$. Both factors likely explain the overall patient preference for PET/CT colonography over colonoscopy. Also, although our regimen was laxativefree, patients did still need to modify their diet. It is likely we were overcautious in this regard, and a truly preparation-free examination is worthy of investigation. We did not attempt to subanalyze the CT colonography and PET portion of the examination given the integral nature of the procedures in clinical practice. In addition, we gave patients no indication of the relative diagnostic performance of the 2 tests, and as such our analysis was based on their physical and emotional experience only (22).

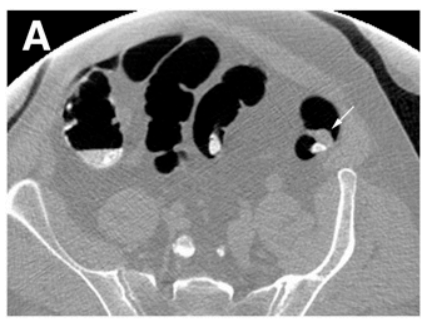

\section{B}

FIGURE 2. Axial CT (A), PET (B), and fused PET/CT colonography (C) images demonstrating 10-mm sigmoid pedunculated tubulovillous adenoma (arrow), which was ${ }^{18} \mathrm{~F}$-FDG-avid (SUV, 7). Arrowhead = ureteric ${ }^{18}$ F-FDG activity.

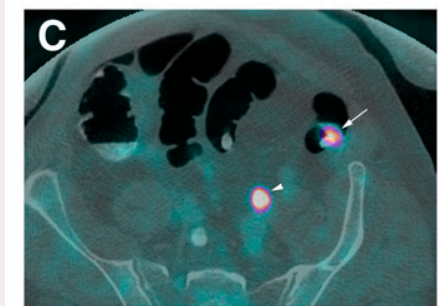

Studies investigating the diagnostic performance of nonlaxative CT colonography alone have produced variable results. Data suggest that use of iodine-based tagging $(8,23)$ may be superior to barium regimens (7), although the laxative effects of ionic-iodinated compounds can be significant and we deliberately chose oral barium because of the known tagging efficiency and minimal side effect profile. Nonlaxative CT (without colonic distension) misses $20 \%$ of tumors, with a PPV of less than 50\% (24). Although the utility of PET for detecting recurrent colorectal malignancy is established (25), its role in primary diagnosis is uncertain. In a recent retrospective study of 110 asymptomatic individuals, $61 \%$ of 23 adenomas $10 \mathrm{~mm}$ or larger were visible in retrospect as ${ }^{18} \mathrm{~F}-\mathrm{FDG}$-avid foci, although lesions $9 \mathrm{~mm}$ or less were apparently undetectable (26). In
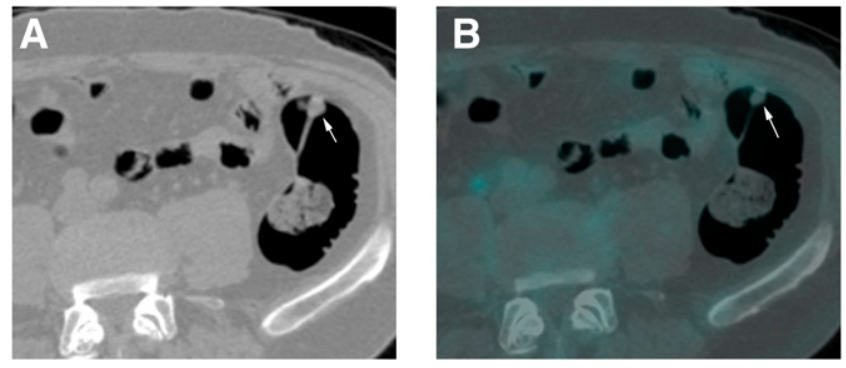

FIGURE 3. Axial CT (A) and fused PET/CT colonography (B) images demonstrating 7-mm sigmoid pedunculated tubulovillous adenoma (arrow), which showed no ${ }^{18} \mathrm{~F}-\mathrm{FDG}$ avidity. 


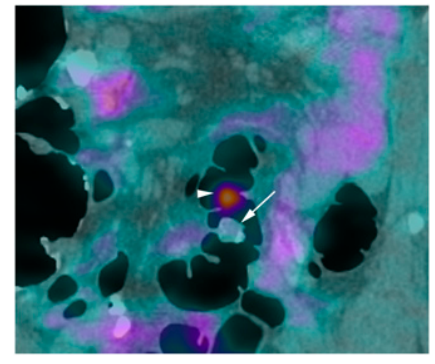

FIGURE 4. Coronal fused $\mathrm{PET} / \mathrm{CT}$ colonography image demonstrating $20-\mathrm{mm}$ sigmoid tubulovillous adenoma (arrow). Polyp is apparently non- ${ }^{18} \mathrm{~F}-\mathrm{FDG}$-avid because of 11-mm misregistration with intense ${ }^{18} \mathrm{~F}$ FDG avidity (arrowhead) (SUV, 15.4).

a smaller 39-patient study, PET had a sensitivity of $74 \%$ and specificity of $84 \%$ for colonic neoplasia, compared with colonoscopy, although again PET failed to detect small (diameter, 3-10 $\mathrm{mm}$ ) polyps in 4 patients (27).

By combining the 2 techniques, we hypothesized that diagnostic performance would increase. In line with standard practice, we investigated the combined PET/CT colonography dataset after first reviewing the CT colonography data. Therefore, we did not directly test a concurrent PET/CT colonography reading paradigm.

Reassuringly, PET/CT colonography detected all polyps $10 \mathrm{~mm}$ or larger, and all were ${ }^{18} \mathrm{~F}-\mathrm{FDG}-$ avid. However, only half those that were $6-9 \mathrm{~mm}$ and no polyps that were $5 \mathrm{~mm}$ or smaller were ${ }^{18} \mathrm{~F}-\mathrm{FDG}$-avid. In a highly enriched cohort of 17 patients undergoing PET/CT colonography after full bowel preparation, Gollub et al. (12) reported ${ }^{18} \mathrm{~F}-$ FDG avidity in $13 \%, 35 \%$, and $59 \%$ of polyps sized $1-5$ $\mathrm{mm}, 6-9 \mathrm{~mm}$, and at least $10 \mathrm{~mm}$, respectively. However, in a small feasibility study, Mainenti et al. (13) reported ${ }^{18} \mathrm{~F}-$ FDG avidity in none of 12 polyps $9 \mathrm{~mm}$ or smaller but in all of the 5 polyps $10 \mathrm{~mm}$ or larger. These data suggest that polyps $10 \mathrm{~mm}$ or larger are often ${ }^{18} \mathrm{~F}-\mathrm{FDG}$-avid, whereas those below the 10-mm threshold are frequently not. In our study, the PET pixel size was a relatively large $5.47 \mathrm{~mm}$. Our scan resolution is in contrast to the studies of Gollub et al. (12) and Mainenti et al. (13), in which the stated inplane resolution was below 3 and $4.2 \mathrm{~mm}$, respectively. This difference in spatial resolution may have influenced our results for smaller polyps. However, larger polyps may be clearly visible on the CT component but show no ${ }^{18} \mathrm{~F}$ FDG uptake, suggesting that factors other than size influence the ${ }^{18} \mathrm{~F}-\mathrm{FDG}$ avidity. Interestingly, in the current study the average SUV of adenomas with high-grade

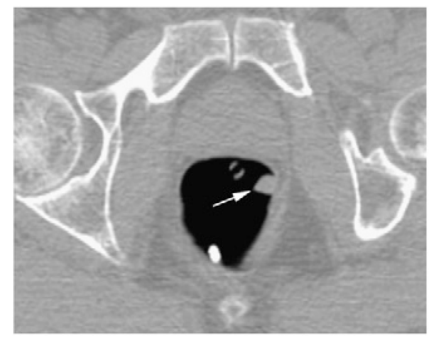

FIGURE 5. Axial CT colonography image showing 10-mm rectal filling defect (arrow) reported as polyp on CT colonography data alone. Lesion is non- ${ }^{18} \mathrm{~F}$ FDG-avid and was correctly dismissed as untagged fecal residue at combined PET/ CT colonography consensus review.

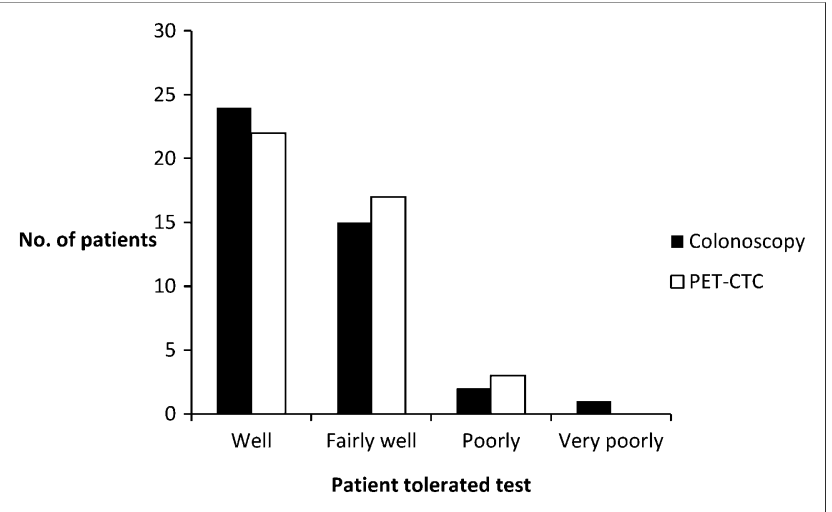

FIGURE 6. Bar chart illustrating patients' responses to question, "I felt I tolerated the test...."

dysplasia was greater than that of adenomas with moderateor low-grade dysplasia, although the difference was not statistically significant, possibly reflecting low power in this pilot study of diagnostic performance. It would be interesting to correlate ${ }^{18} \mathrm{~F}-\mathrm{FDG}$ avidity with genetic mutations with the adenoma-carcinoma pathway and test alternative tracers such as ${ }^{18} \mathrm{~F}$-thymidine, uptake of which is related to cell proliferation in colorectal cancer (28). All the avid polyps in our study had an SUV of greater than 3.4, and although recent work has shown reasonable reproducibility of SUV measurements in gastrointestinal malignancy (29), it is debatable how clinically useful quantitative measurement of uptake will be given the relative small size of polyps and variability in background physiologic bowel activity. Indeed, evidence documenting the major influence of partial voluming in the SUV measurement of small lesions is emerging (30).

The PET component did not improve sensitivity over that of CT colonography, but the radiologist was familiar with CT colonography interpretation. It would be interesting to investigate less experienced radiologists - it is possible that PET activity would aid polyp detection. Indeed, the use of a single reading physician would be more efficient, and the

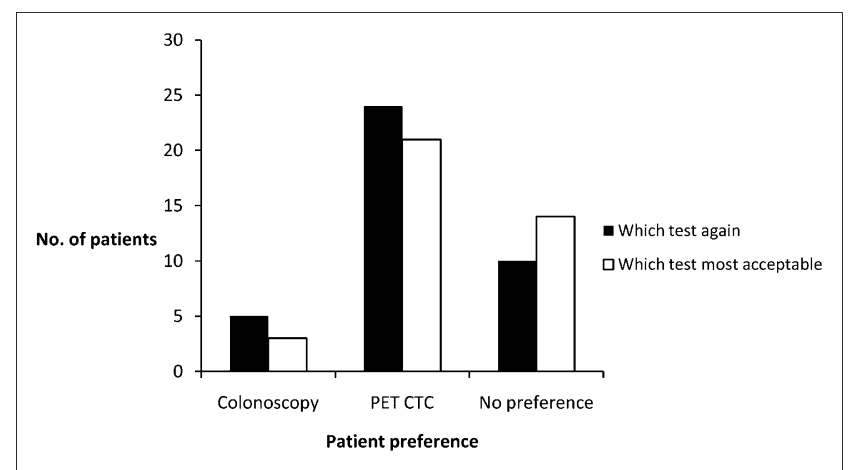

FIGURE 7. Bar chart illustrating patient's preferences for future repeated testing and overall test preference. 
benefits of PET could be greater for a single reader. However, double reading of standard CT colonography or use of computer-aided diagnosis are arguably simpler strategies to improve reader performance $(31,32)$, although neither approach is infallible.

Conversely, the PET component had an important benefit in terms of overall specificity and diagnostic confidence. The PPV of combined PET/CT colonography for patients with polyps $10 \mathrm{~mm}$ or larger was $27 \%$ greater than for CT colonography. Specifically, CT colonography alone would have triggered an extra 3 colonoscopies due to falsepositive lesions measuring greater than $10 \mathrm{~mm}$. Furthermore, diagnostic confidence that the colon was normal also improved with PET, and attenuation-correction artifacts from retained high-density barium were not problematic. Perhaps unexpectedly, we did not find that misregistration between PET avidity and CT colonography abnormality was a major problem, despite differences in resolution between the 2 techniques and potential difference in bowel distension between PET and CT colonography acquisitions. Although we have limited polyp numbers in this pilot study, the data are reassuring. We did not formally assess how well colonic distension was maintained during the PET acquisition, but we aimed to maintain good distension throughout using constant low-volume $\mathrm{CO}_{2}$ colonic insufflation. Anecdotally, we did not see a great change, and the presence of good registration for most polyps suggests distension was maintained, although this is an area for further study. Of course the improvement in specificity and diagnostic confidence with PET turns on the reader assumption that significant lesions would show ${ }^{18} \mathrm{~F}-\mathrm{FDG}$ avidity.

An additional benefit of cross-sectional imaging in older symptomatic patients is the ability to assess extracolonic organs: we detected an unsuspected gastric tumor, for example. Furthermore, the additional specificity afforded by PET potentially has beneficial effects on cost-effectiveness. In the current study, an indeterminate adrenal mass and presacral soft-tissue thickening were both non- ${ }^{18} \mathrm{~F}$ FDG-avid and subsequently proven benign on further work-up, which was arguably unnecessary. As noted by others, if a colonic tumor is detected, PET/CT colonography can both stage the primary tumor and assess the rest of the colon $(15,33)$.

Although our data are promising, the role of nonlaxative PET/CT colonography needs further definition. Clearly PET/CT colonography is expensive, and the current application will likely be reserved for patients intolerant of full bowel laxation in whom a sensitive and highly specific test is required to exclude colonic pathology. It is difficult to justify its use just to increase sensitivity for smaller lesions when recent data using iodine-based tagging, perhaps with computer-aided detection, suggest standard CT colonography achieves adequate sensitivity $(23,32)$. Furthermore, the clinical significance of smaller polyps in an older, frailer population is debatable anyway, and even the prevalence of advanced neoplasia in these patients is relatively low. However, as discussed, standard unprepared CT has a PPV of just $50 \%$ (24), resulting in unnecessary and potentially dangerous investigations in $50 \%$ of those who test positive. Also, the recent American College of Radiology Imaging Network 2 study reported a PPV of just $23 \%$ for cancer or for polyps larger than $1 \mathrm{~cm}$, even with full bowel preparation and tagging (34). In contrast, we found the PPV of PET/CT colonography reassuringly high, albeit in our small pilot study of diagnostic performance. It could, therefore, be that PET/CT colonography should be reserved for patients with a high underlying clinical suggestion of neoplasia (colonic and perhaps extracolonic) in whom any laxation is ideally avoided. A normal PET/CT colonography result would be highly reassuring, thus obviating further investigations. Conversely, a positive result would be sufficiently concerning to justify more invasive tests. Another role for PET/CT colonography may be for staging patients with known colonic cancer after incomplete colonoscopy (33). Finally, PET/CT colonography may allow us to learn more about the spectrum of pathologies in the polypadenoma sequence. For example, the pathologic significance of serrated adenomas and methylation pathways remains poorly understood and ${ }^{18}$ F-FDG avidity could produce future insights.

Our study does have limitations. Although the largest consecutive series to date, our cohort remains necessarily small. However, we were able to demonstrate the technical feasibility and patient preference for PET/CT colonography over colonoscopy (which were our main aims) and benefits to reader confidence. We had few clinically significant lesions in our cohort, but the prevalence of abnormality was typical of patients undergoing colonoscopy in an outpatient setting, and our test of diagnostic performance was in reality a pilot. All of our patients had to be fit enough to undergo subsequent bowel purgation and colonoscopy. Our technique must now be tested in those who are frailer. Although we feel it was important to use highly experienced readers in this initial proof-of-concept study, further work is required to assess performance using less experienced readers. To reduce overall radiation exposure, our protocol utilized half the standard ${ }^{18} \mathrm{~F}-\mathrm{FDG}$ dose, which could have reduced the ability to detect the avidity of smaller polyps and also increased PET acquisition time. Arguably, the need to limit radiation exposure is of much less concern in older patients, when a quicker efficient examination is the priority.

\section{CONCLUSION}

Simultaneous PET acquisition during nonlaxative CT colonography is technically feasible; is well tolerated by patients; and, in this pilot study, potentially improves diagnostic specificity, confidence, and PPV, although not sensitivity. Use of this specialized technology will likely be reserved for patients intolerant of full bowel laxation in 
whom a sensitive and highly specific test is required to exclude significant colonic pathology.

\section{ACKNOWLEDGMENTS}

We thank Gina Cattini, Dr. Stuart Bloom, and Austin Obichere for helping with data collection. This work was undertaken at UCLH/UCL, which received a proportion of funding from the Department of Health's NIHR Biomedical Research Centres funding scheme. A portion of this study was funded by grant support from the Clinical Research and Development Committee, UCLH Charities, and the Royal Free and University College Medical School. The views expressed in this publication are those of the authors and not necessarily those of the Department of Health.

\section{REFERENCES}

1. Sosna J, Morrin MM, Kruskal JB, Lavin PT, Rosen MP, Raptopoulos V. CT colonography of colorectal polyps: a metaanalysis. AJR. 2003;181:1593-1598.

2. Steffes CP, Sugawa C, Wilson RF, Hayward SR. Oxygen saturation monitoring during endoscopy. Surg Endosc. 1990;4:175-178.

3. Flashman K, O'Leary DP, Senapati A, Thompson MR. The Department of Health's "two week standard" for bowel cancer: is it working? Gut. 2004;53: 387-391.

4. Ristvedt SL, McFarland EG, Weinstock LB, Thyssen EP. Patient preferences for CT colonography, conventional colonoscopy, and bowel preparation. Am J Gastroenterol. 2003;98:578-585.

5. Heymann TD, Chopra K, Nunn E, Coulter L, Westaby D, Murray-Lyon IM. Bowel preparation at home: prospective study of adverse effects in elderly people. BMJ. 1996;313:727-728.

6. Callstrom MR, Johnson CD, Fletcher JG, et al. CT colonography without cathartic preparation: feasibility study. Radiology. 2001;219:693-698.

7. Johnson CD, Manduca A, Fletcher JG, et al. Noncathartic CT colonography with stool tagging: performance with and without electronic stool subtraction. AJR. 2008; 190:361-366.

8. Iannaccone R, Laghi A, Catalano C, et al. Computed tomographic colonography without cathartic preparation for the detection of colorectal polyps. Gastroenterology. 2004;127:1300-1311.

9. Liedenbaum MH, de Vries AH, Gouw CI, et al. CT colonography with minimal bowel preparation: evaluation of tagging quality, patient acceptance and diagnostic accuracy in two iodine-based preparation schemes. Eur Radiol. 2010;20:367-376.

10. Israel O, Yefremov N, Bar-Shalom R, et al. PET/CT detection of unexpected gastrointestinal foci of ${ }^{18} \mathrm{~F}-\mathrm{FDG}$ uptake: incidence, localization patterns, and clinical significance. J Nucl Med. 2005;46:758-762.

11. Kayani I, Groves AM, Syed R, Bomanji J. Combined F-18 FDG positron emission tomography/computed tomography in the diagnosis of colonic polyps: the potential and limitations of the technique. Clin Nucl Med. 2005;30: 116-117.

12. Gollub MJ, Akhurst T, Markowitz AJ, et al. Combined CT colonography and ${ }^{18}$ F-FDG PET of colon polyps: potential technique for selective detection of cancer and precancerous lesions. AJR. 2007;188:130-138.
13. Mainenti PP, Salvatore B, D'Antonio D, et al. PET/CT colonography in patients with colorectal polyps: a feasibility study. Eur J Nucl Med Mol Imaging. 2007; 34:1594-1603.

14. Veit-Haibach P, Kuehle CA, Beyer T, et al. Diagnostic accuracy of colorectal cancer staging with whole-body PET/CT colonography. JAMA. 2006;296:2590-2600.

15. Veit $\mathrm{P}$, Kuhle $\mathrm{C}$, Beyer $\mathrm{T}$, et al. Whole body positron emission tomography/ computed tomography (PET/CT) tumour staging with integrated PET/CT colonography: technical feasibility and first experiences in patients with colorectal cancer. Gut. 2006;55:68-73.

16. Dachman AH. Advice for optimizing colonic distention and minimizing risk of perforation during CT colonography. Radiology. 2006;239:317-321.

17. Zalis ME, Barish MA, Choi JR, et al. CT colonography reporting and data system: a consensus proposal. Radiology. 2005;236:3-9.

18. O'Brien MJ, Winawer SJ, Zauber AG, et al. The National Polyp Study. Patient and polyp characteristics associated with high-grade dysplasia in colorectal adenomas. Gastroenterology. 1990;98:371-379.

19. Pickhardt PJ, Choi JR, Hwang I, et al. Computed tomographic virtual colonoscopy to screen for colorectal neoplasia in asymptomatic adults. $N$ Engl J Med. 2003;349:2191-2200.

20. Salmon P, Shah R, Berg S, Williams C. Evaluating customer satisfaction with colonoscopy. Endoscopy. 1994;26:342-346.

21. Holemans JA, Matson MB, Hughes JA, Seed P, Rankin SC. A comparison of air, $\mathrm{CO} 2$ and an air/CO2 mixture as insufflation agents for double contrast barium enema. Eur Radiol. 1998;8:274-276.

22. von WC. Halligan S, Atkin WS, Lilford RJ, Morton D, Wardle J. Choosing between CT colonography and colonoscopy in the diagnostic context: a qualitative study of influences on patient preferences. Health Expect. 2009;12:18-26.

23. Liedenbaum MH, de Vries AH, van Rijn AF, et al. CT colonography with limited bowel preparation for the detection of colorectal neoplasia in an FOBT positive screening population. Abdom Imaging. November 4, 2009 [Epub ahead of print].

24. Ng CS, Doyle TC, Pinto EM, et al. Evaluation of CT in identifying colorectal carcinoma in the frail and disabled patient. Eur Radiol. 2002;12:2988-2997.

25. Whiteford MH, Whiteford HM, Yee LF, et al. Usefulness of FDG-PET scan in the assessment of suspected metastatic or recurrent adenocarcinoma of the colon and rectum. Dis Colon Rectum. 2000;43:759-767.

26. Yasuda S, Fujii H, Nakahara T, et al. ${ }^{18}$ F-FDG PET detection of colonic adenomas. J Nucl Med. 2001;42:989-992.

27. Drenth JP, Nagengast FM, Oyen WJ. Evaluation of (pre-)malignant colonic abnormalities: endoscopic validation of FDG-PET findings. Eur J Nucl Med. 2001;28:1766-1769.

28. Francis DL, Freeman A, Visvikis D, et al. In vivo imaging of cellular proliferation in colorectal cancer using positron emission tomography. Gut. 2003;52:1602-1606.

29. Velasquez LM, Boellaard R, Kollia G, et al. Repeatability of ${ }^{18}$ F-FDG PET in a multicenter phase I study of patients with advanced gastrointestinal malignancies. J Nucl Med. 2009;50:1646-1654.

30. Tylski P, Stute S, Grotus N, et al. Comparative assessment of methods for estimating tumor volume and standardized uptake value in ${ }^{18} \mathrm{~F}-\mathrm{FDG}$ PET. $J$ Nucl Med. 2010;51:268-276.

31. Johnson CD, Harmsen WS, Wilson LA, et al. Prospective blinded evaluation of computed tomographic colonography for screen detection of colorectal polyps. Gastroenterology. 2003;125:311-319.

32. Baker ME, Bogoni L, Obuchowski NA, et al. Computer-aided detection of colorectal polyps: can it improve sensitivity of less-experienced readers? Preliminary findings. Radiology. 2007;245:140-149.

33. Nagata K, Ota Y, Okawa T, Endo S, Kudo SE. PET/CT colonography for the preoperative evaluation of the colon proximal to the obstructive colorectal cancer. Dis Colon Rectum. 2008;51:882-890.

34. Johnson CD, Chen MH, Toledano AY, et al. Accuracy of CT colonography for detection of large adenomas and cancers. N Engl J Med. 2008;359:1207-1217. 